\title{
Two new pulsating low-mass pre-white dwarfs or SX Phoenicis stars?^
}

\author{
M. A. Corti ${ }^{1,2}$, A. Kanaan ${ }^{3}$, A. H. Córsico ${ }^{1,4}$, S. O. Kepler ${ }^{5}$, L. G. Althaus ${ }^{1,4}$, D. Koester ${ }^{6}$, and J. P. Sánchez Arias ${ }^{1,4}$ \\ ${ }^{1}$ Grupo de Evolución Estelar y Pulsaciones, Facultad de Ciencias Astronómicas y Geofísicas, Universidad Nacional de La Plata, \\ Paseo del Bosque s/n, 1900 La Plata, Argentina \\ e-mail: mariela@fcaglp.unlp.edu.ar \\ 2 Instituto Argentino de Radioastronomía, CCT-La Plata, CONICET, C.C. Nro. 5, 1984 Villa Elisa, Argentina \\ ${ }^{3}$ Departamento de Física, Centro de Ciências Físicas e Matemáticas, Universidade Federal de Santa Catarina, \\ Campus Universitário Reitor João David Ferreira Lima, Florianópolis, Brazil \\ 4 Instituto de Astrofísica La Plata, CONICET-UNLP, Paseo del Bosque s/n, 1900 La Plata, Argentina \\ 5 Departamento de Astronomia, Universidade Federal do Rio Grande do Sul, Av. Bento Gonçalves 9500, 91501-970 Porto Alegre, \\ RS, Brazil \\ ${ }^{6}$ Institut für Theoretische Physik und Astrophysik, Universität Kiel, 24098 Kiel, Germany
}

Received 28 September 2015 / Accepted 4 February 2016

\section{ABSTRACT}

\begin{abstract}
Context. The discovery of pulsations in low-mass stars opens an opportunity to probe their interiors and determine their evolution by employing the tools of asteroseismology.

Aims. We aim to analyse high-speed photometry of SDSS J145847.02+070754.46 and SDSS J173001.94+070600.25 and discover brightness variabilities. In order to locate these stars in the $T_{\text {eff }}-\log g$ diagram, we fit optical spectra (SDSS) with synthetic nonmagnetic spectra derived from model atmospheres.

Methods. To carry out this study, we used the photometric data we obtained for these stars with the $2.15 \mathrm{~m}$ telescope at CASLEO, Argentina. We analysed their light curves and applied the discrete Fourier transform (FT) to determine the pulsation frequencies. Finally, we compare both stars in the $T_{\text {eff }}-\log g$ diagram, with two known pre-white dwarfs and seven pulsating pre-ELM white dwarf stars, $\delta$ Scuti, and SX Phe stars

Results. We report the discovery of pulsations in SDSS J145847.02+070754.46 and SDSS J173001.94+070600.25. We determine their effective temperature and surface gravity to be $T_{\text {eff }}=7972 \pm 200 \mathrm{~K}, \log g=4.25 \pm 0.5$ and $T_{\text {eff }}=7925 \pm 200 \mathrm{~K}, \log g=4.25 \pm$ 0.5 , respectively. With these parameters, these new pulsating low-mass stars can be identified with either ELM white dwarfs (with $\sim 0.17 M_{\odot}$ ) or more massive SX Phe stars. We identified pulsation periods of 3278.7 and $1633.9 \mathrm{~s}$ for SDSS J145847.02+070754.46 and a pulsation period of $3367.1 \mathrm{~s}$ for SDSS J173001.94+070600.25. These two new objects, together with those of Maxted et al. (2013, 2014), indicate the possible existence of a new instability domain towards the late stages of evolution of low-mass white dwarf stars, although their identification with SX Phe stars cannot be discarded.
\end{abstract}

Key words. white dwarfs - stars: individual: SDSSJ145847.02+070754.46 - stars: individual: SDSSJ173001.94+070600.25 stars: low-mass

\section{Introduction}

Low-mass $\left(M_{\star} / M_{\odot} \lesssim 0.45\right)$ white dwarfs (WDs) are thought to be the outcome of strong mass-loss events at the red giant branch stage of low-mass stars in binary systems before the He flash onset (Althaus et al. 2010). Since the He flash does not occur, their cores must be made of He. This is in contrast to the case of average-mass $\left(M_{\star} \sim 0.6 M_{\odot}\right)$ WDs, which are thought to have $\mathrm{C} / \mathrm{O}$ cores. Binary evolution is the most likely origin for the so-called extremely low-mass (ELM) WDs, which have masses below $\sim 0.18-0.20 M_{\odot}$. It is well known (Driebe et al. 1998) that ELM WDs are expected to harbour very thick H envelopes, which are able to sustain residual $\mathrm{H}$ nuclear burning via $p p$-chain, leading to markedly long evolutionary timescales. Numerous low-mass WDs, including ELM WDs, have been detected through the ELM survey and the SPY and WASP surveys (Koester et al. 2009; Brown et al. 2010, 2012, 2013;

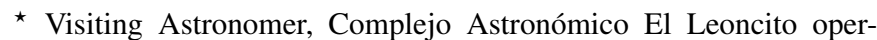
ated under agreement between the Consejo Nacional de Investigaciones Científicas y Técnicas de la República Argentina and the National Universities of La Plata, Córdoba, and San Juan.
Maxted et al. 2011; Kilic et al. 2011, 2012; Gianninas et al. 2015). The interest in low-mass WDs has been greatly boosted by the discovery that some of them pulsate (ELMVs; Hermes et al. 2012, 2013a,b; Kilic et al. 2015; Bell et al. 2015), which constitutes an unprecedented opportunity to probe their interiors and eventually to test their formation channels by employing the tools of asteroseismology.

High-frequency pulsations in a precursor of a low-mass WD star component of an eclipsing binary system were reported by Maxted et al. (2013). The object analysed by those authors, 1SWASP J024743.37-251549.2B (hereinafter WASP J0247-25B, $\log g=4.58, T_{\text {eff }}=11380 \mathrm{~K}, M_{\star}=$ $\left.0.186 M_{\odot}\right)$, showed variability with periods in the range $380-420 \mathrm{~s}$, which is likely due to a mixture of radial $(\ell=0)$ and nonradial $(\ell \geq 1) p$-modes. Theoretical models predict that this star is evolving to higher effective temperatures at nearly constant luminosity prior to becoming a low-mass WD. This could be the first member of a new class of pulsating stars that are the precursors of low-mass WDs. A second object of this type, 1SWASP J162842.31+101416.7B (hereinafter WASP J1628+10B; $\log g=4.49, T_{\text {eff }}=9200 \mathrm{~K}$, 
$M_{\star}=0.135 M_{\odot}$ ), was discovered by Maxted et al. (2014) in another eclipsing binary system, showing high-frequency signals likely to be due to pulsations similar to those seen in WASP J0247-25B. However, additional photometry is required to confirm that the high-frequency variations are due to pulsations in the pre-WD star, and not produced by the companion star, a normal A2V star showing $\delta$ Scuti-type pulsations. Shortly after the discovery of the short-period oscillations in WASP J0247-25B, Jeffery \& Saio (2013) explored the pulsation stability of radial modes of low-mass WD models considering a range of envelope chemical composition, effective temperature, and luminosity. Jeffery \& Saio (2013) identified the instability boundaries associated with radial modes characterized by low-to-high radial orders, and showed that they are very sensitive to composition. These authors found that the excitation of modes is by the $\kappa$-mechanism operating mainly in the second He-ionization zone, provided that the driving region is depleted in $\mathrm{H}\left(0.2 \lesssim X_{\mathrm{H}} \lesssim 0.3\right)$.

The domain of the precursors of ELM WDs in the $T_{\text {eff }}-\log g$ plane could be coincident with that corresponding to the pulsating $\delta$ Scuti or SX Phe stars. It is not possible to distinguish between both classes of pulsating stars by amplitude and number of frequencies. There are several SX Phe stars with characteristics similar to the high-amplitude $\delta$ Scuti stars except that field SX Phe have high proper motions and low metallicity (Fauvaud et al. 2010). SX Phe stars probably belong to the thick disc or halo and may therefore be considered to be in an advanced stage of evolution, while the $\delta$ Scuti are main-sequence stars. The SX Phe stars that are not field stars are to be found in globular clusters (Balona \& Nemec 2012).

The members of both SX Phe and $\delta$ Scuti classes of variable stars have $1.5-2.3 M_{\odot}$ and exhibit pulsations due to low-order radial and nonradial $p$-modes with periods between $12 \mathrm{~min}$ to $10 \mathrm{~h}$ (Catelan \& Smith 2015). Most SX Phe and $\delta$ Scuti stars have $6000<T_{\text {eff }}<9000 \mathrm{~K}$ and a logarithm of gravities of around 4.0. However, some of these stars are hotter than the average, thus populating the same region of the $T_{\text {eff }}-\log g$ diagram where precursors of ELM WDs could be located.

In this paper, we report on the discovery of SDSS J173001.94+070600.25 $\left(T_{\text {eff }}=7972 \pm 200 \mathrm{~K}\right.$, $\log g=4.25 \pm 0.5)$ and SDSS J145847.02+070754.46 $\left(T_{\text {eff }}=7925 \pm 200 \mathrm{~K}, \log g=4.25 \pm 0.5\right)$, two new pulsating low-mass stars, which could be precursors of ELM WDs or instead, SX Phe stars. In Sects. 2 and 3, we present the observational material and analysis of the data, respectively. In Sect. 4 we discuss the evolutionary status of these new pulsating stars and give a brief conclusion.

\section{Data}

The observational material consists of high-speed photometry of the $\mathrm{J} 1458+0707$ and $\mathrm{J} 1730+0706$ stars obtained in 2015 during the nights of April 24th to 26th. We used a TEK $1024 \times$ 1024 CCD with pixel size of $24 \mu \mathrm{m}$, binned by a factor 2 , attached to the $2.15 \mathrm{~m}$ telescope at Complejo Astronómico El Leoncito (CASLEO) ${ }^{1}$ in San Juan, Argentina. Observations were obtained through a red cut-off $3 \mathrm{~mm}$ BG40 filter to reduce sky background.

We obtained a total of 156 frames of J1458+0707 during the two first nights (119 the first and 37 the second night) and 89 frames of J1730+0706 during the last night. The exposure

\footnotetext{
1 Operated under agreement between CONICET, SeCyT, and the Universities of La Plata, Córdoba, and San Juan, Argentina.
}

time for each image was $120 \mathrm{~s}$, the seeing averaged $2 \mathrm{~s}$ of arc, and the second night was cut short by clouds. Bias, dark, and flat-field frames were obtained every night. All images were obtained by employing the data acquisition programme ROPER and these were analysed applying aperture photometry with the external IRAF package $c c d_{\mathrm{hsp}}$ (Kanaan et al. 2002).

\section{Analysis}

SDSS J1458+0707 and SDSS J1730+0706 were classified as A stars by the ELODIE pipeline of DR12 of SDSS. As part of our pulsating ELM candidate selection programme, we fitted the optical spectra to pure hydrogen local thermodynamic equilibrium (LTE) grids of synthetic non-magnetic spectra derived from model atmospheres (Koester 2010). The model grid uses the ML2 $/ \alpha=0.8$ approximation. Our grid covers effective temperature and gravity values in the ranges $6000 \mathrm{~K} \leq T_{\text {eff }} \leq 100000 \mathrm{~K}$, and $3.75 \leq \log g \leq 10.0$, respectively, covering main-sequence, subdwarfs, and white dwarf spectra.

We fitted the SDSS spectral lines with models that work well in the white dwarf regime above $\log g \sim 5.50$. Adjusting the spectral lines with lower gravity models becomes increasingly difficult because the degeneracy of the parameters becomes very significant. A change in surface gravity can almost completely be compensated by a change in temperature. The moderate resolution of the SDSS spectra is another problem. The higher Balmer lines become narrower at low surface gravity and are thus strongly affected by the instrumental profile. On the other hand, the SDSS photometry is very useful to lift this degeneracy, mainly because of the highly gravity sensitive Balmer jump measured by $u-g$ colour. We therefore started the analysis with a fit of the photometry with theoretical values. The possible reddening is not a severe problem; the objects are very far outside the galactic plane. Assuming a fixed $\log g$ between 3.75 and 4.75 the best fitting temperature is almost constant. As a rather conservative estimate we use a result of the photometry fitting the values in Table 1. The calcium abundance was determined from a comparison of theoretical line strengths with the observed spectra. The errors also include the complete range of errors of the parameters.

\subsection{SDSS J1458+0707}

To build the light curve shown in Fig. 1, we determined the aperture that provides the light curves with minimum scatter. The optimum aperture radius was 12 pixels. The sky annulus used for sky subtraction had an inner radius of 20 pixels and 6 pixels width. The light curve for the pulsating star and a comparison star of similar intensity are shown in Fig. 1. Both stars were divided by the same comparison star, 0.4 mag brighter, to correct for atmospheric transparency fluctuations. Each point in the light curves was normalized by the average of all light curve points. The variations visible in the comparison star light curve are attributed to noise.

We computed the FT of the data from the two nights, joining the two light curves after correcting the timings to the Barycentric Julian Ephemeris Date (IAU SOFA). To check for sampling artifacts we computed a spectral window as well. Figure 2 shows the FT and the spectral window. The main peak in the FT is labelled $f_{2}$ and corresponds to the variations clearly seen in the light curve. The small peak $f_{1}$ has an analog in the spectral window at the same frequency and same intensity relative to the $f_{2}$, we dismiss $f_{1}$ as an alias peak. Another frequency with intensity above the $3.3\langle\mathrm{~A}\rangle$ noise limit (Kepler 1993) is $f_{3}$. It is probably an $f_{2}$ harmonic because it is almost twice $f_{2}$. 

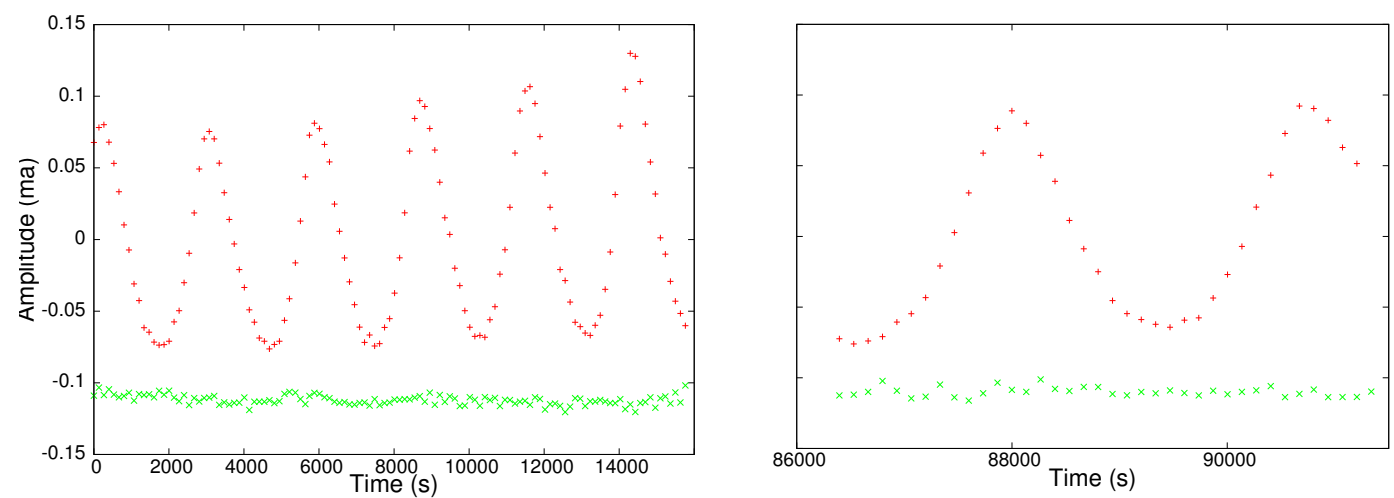

Fig. 1. Top curve: high-speed photometry of J1458+0707 star over data points obtained during two consecutive nights. Bottom curve: time variations of the brightest comparison star in the field over the same period (see text and Table 1).

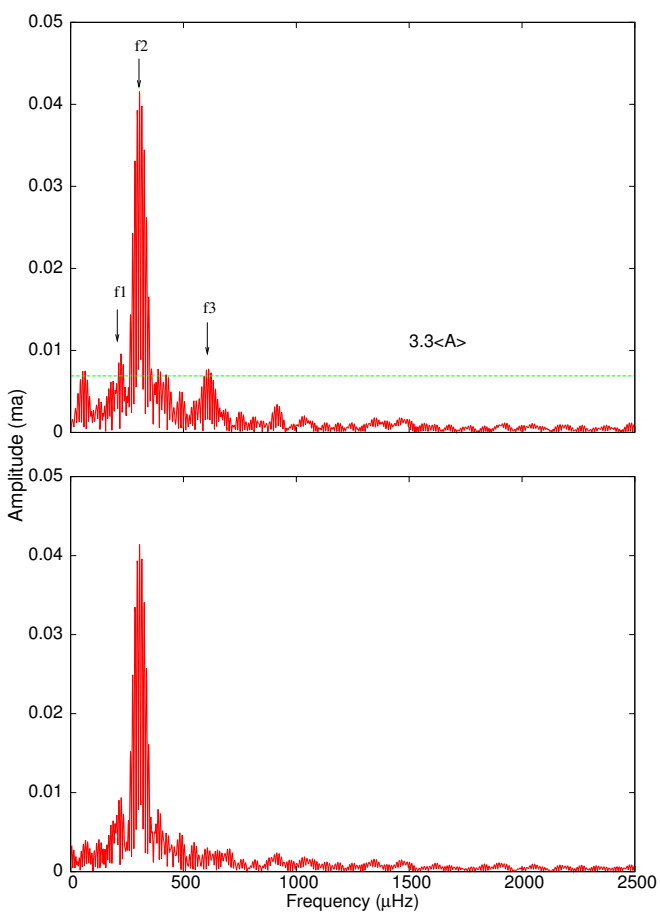

Fig. 2. Top panel: discrete FT of the light curve of J1458+0707. The horizontal line shows 3.3 times the noise level. There are three pulsation frequencies (see text and Table 1). Bottom panel: spectral window with more intense emission peak at $305 \mu \mathrm{Hz}$.

\subsection{SDSS J1730+0706}

This star and its comparison stars were analysed in the same manner as J1458+0707. The top light curve shown in Fig. 3 corresponds to $\mathrm{J} 1730+0706$ divided by a comparison star that is $1 \mathrm{mag}$ brighter. The bottom light curve was obtained for the brightest comparison stars (1.5 mag brighter than the target star) divided by the same comparison star used for the target object. The peak-to-peak variations in this last light curve reach $0.01 \mathrm{ma}$, which is totally attributable to noise.

We employed the same Fourier analysis technique as for $\mathrm{J} 1458+0707$, the FT for this object is shown in Fig. 4. with an average modulation amplitude $\langle A\rangle=3.0 \mathrm{mma}$ (Kepler 1993). The parameters for the only significant peak are shown in Table 1.

\section{Discussion and conclusions}

The seven pulsating ELM WD stars known (Hermes et al. 2012, 2013a,b; Kilic et al. 2015; Bell et al. 2015) have effective temperatures between $\sim 10000 \mathrm{~K}$ and $\sim 7500 \mathrm{~K}$, and $6<\log g<7$. The

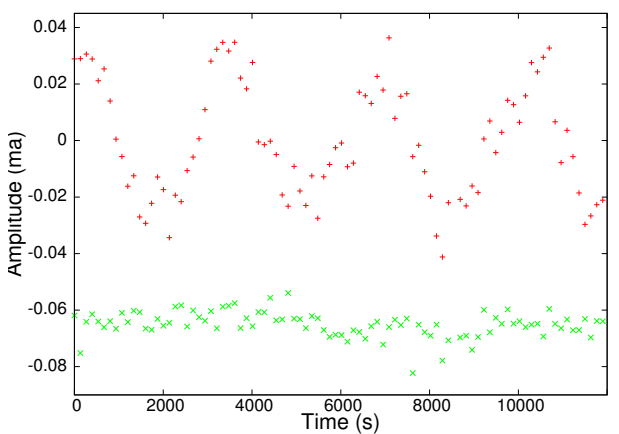

Fig. 3. Top curve: high-speed photometry of J1730+0706 star over data points obtained during one night. Bottom curve: time variations of the brightest comparison star in the field over the same period (see text and Table 1).

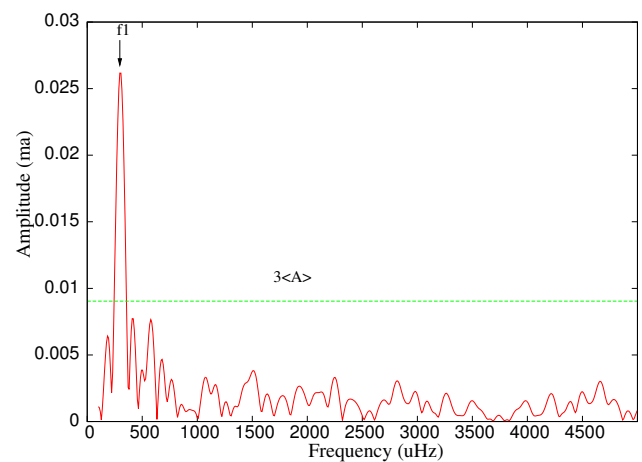

Fig. 4. Discrete FT of the J1730+0706 light curve. The horizontal line shows three times the noise level. There is one pulsation frequency (see text and Table 1).

location of these stars in the $T_{\text {eff }}-\log g$ diagram is depicted in Fig. 5 as light green dots. Also shown are the evolutionary tracks of low-mass He-core WDs as computed by Althaus et al. (2013, dotted black lines) in the range of masses $0.1554-0.2707 M_{\odot}$. For the sake of completeness, we include also a $0.148 M_{\odot}$ evolutionary track computed by Serenelli et al. (2001, dashed black line). In addition, we show with grey and black dots the location of a sample of $\delta$ Scuti stars extracted from Uytterhoeven et al. (2011) and Bradley et al. (2015), respectively, and the location of a sample of SX Phe stars with pink dots, extracted from the Balona \& Nemec (2012). Finally, the locations of SDSS J1730+0706 and SDSS J1458+0707 are indicated with red dots, along with the already known pulsating pre-WD stars WASP J0247-25B and WASP J1628+10B (Maxted et al. 2013, 2014), which are indicated with blue dots. Clearly, given the 
Table 1. Data of pulsating stars.

\begin{tabular}{ccccccccc}
\hline \hline $\begin{array}{c}\text { ID } \\
\text { SDSS }\end{array}$ & $\begin{array}{c}\text { Period } \\
(\mathrm{s})\end{array}$ & $\begin{array}{c}\text { Frequency } \\
(\mu \mathrm{Hz})\end{array}$ & $\begin{array}{c}\text { Amplitude } \\
(\mathrm{ma})\end{array}$ & $\begin{array}{c}T_{\text {eff }} \\
(\mathrm{K})\end{array}$ & $\log g$ & {$[\mathrm{Ca} / \mathrm{H}]$} & $\begin{array}{c}\mathrm{Z} \\
(\mathrm{pc})\end{array}$ & $\begin{array}{c}\mu^{\Delta} \\
\left(\mathrm{mas} \mathrm{yr}^{-1}\right)\end{array}$ \\
\hline $\mathrm{J} 145847.02+070754.46$ & 3278.7 & $\mathrm{f} 2=305$ & 0.042 & $7925 \pm 200$ & $4.25 \pm 0.5$ & $-7.00 \pm 0.25$ & 2559 & $55 \pm 4$ \\
& 1633.9 & $\mathrm{f} 3=612$ & 0.008 & & & & & \\
$\mathrm{~J} 173001.94+070600.25$ & 3367.1 & $\mathrm{f} 1=297$ & 0.026 & $7972 \pm 200$ & $4.25 \pm 0.5$ & $-6.55 \pm 0.25$ & 1745 & $12 \pm 5$ \\
\hline
\end{tabular}

Notes. ${ }^{(\Delta)}$ Zacharias et al. (2012).

very low surface gravities characterizing SDSS J1730+0706 and SDSS J1458+0707 ( $\log g \sim 4.3)$, these two stars cannot be identified with low-mass WDs. Instead, they could be associated with either low-mass pre-WD stars with stellar masses between $\sim 0.17$ and $\sim 0.20 M_{\odot}$ or with the hottest known $\delta$ Scuti or SX Phe stars.

If the stars presented in this work are indeed precursors of low-mass WDs, we can trace their following evolution. Both stars would evolve to hotter effective temperatures directly to their terminal cooling track. We would conclude that these new pulsating stars are of the same type as the pulsating pre-WDs WASP J0247-25B and WASP J1628+10B.

We note, however, that there exists some differences concerning the observed pulsation properties. Indeed, SDSS J1730+0706 and SDSS J1458+0707 pulsate with periods in the range 1634-3367 s, which is longer than those exhibited by WASP J0247-25B and WASP J1628+10B, which show periods in the range 400-800 s (Maxted et al. 2013, 2014). Also, it is apparent that the amplitudes observed in SDSS J1730+0706 and SDSS J1458+0707 (up to 40 mmag) are larger than those detected in WASP J0247-25B and WASP J1628+10B (of the order of a few mmag). According to the theoretical analysis of Jeffery \& Saio (2013), at least the pulsations of WASP J0247-25B should be due to radial modes of high radial order. Instead, the long periods observed in SDSS J1730+0706 and SDSS J1458+0707 are likely due to high-order nonradial $g$ modes. The question of why short periods typical of $p$-modes (or radial modes) are not detected in SDSS J1730+0706 and SDSS J1458+0707, and vice versa, why no long periods characteristic of $g$-modes are observed in WASP J0247-25B and WASP J1628+10B, could be answered through a full (radial and nonradial) linear pulsation stability analysis. This analysis will be addressed in a future investigation. Concerning the contrast in the amplitudes of $g$-modes of SDSS J1730+0706 and SDSS J1458+0707 as compared with those of the $p$-modes exhibited by WASP J0247-25B and WASP J1628+10B, a definitive answer could be given by non-linear pulsation calculations, which are out the scope of this paper.

If instead, these stars with metallicity lower than solar metallicity and with proper motion of the stars in the galaxy halo were SX Phe stars, they would populate the hottest portion of the SX Phe instability strip. Reversing the argument, after examining Fig. 5, we cannot discard the possibility that some of the hottest known SX Phe stars might otherwise be low-mass pre-WD stars.

Acknowledgements. M.A.C. acknowledge support from CONICET (PIP 112201201-00226). A.K. and S.O.K. acknowledge CNPq support. D.K. received support from programme Science without Borders, MCIT/MEC-Brazil. We thank Dr. Costa José Eduardo for allowing us to use his DFT programme. We wish to thank the anonymous referee for the suggestions and comments that improved the original version of this work.

\section{References}

Althaus, L. G., Córsico, A. H., Isern, J., \& García-Berro, E. 2010, A\&ARv, 18, 471

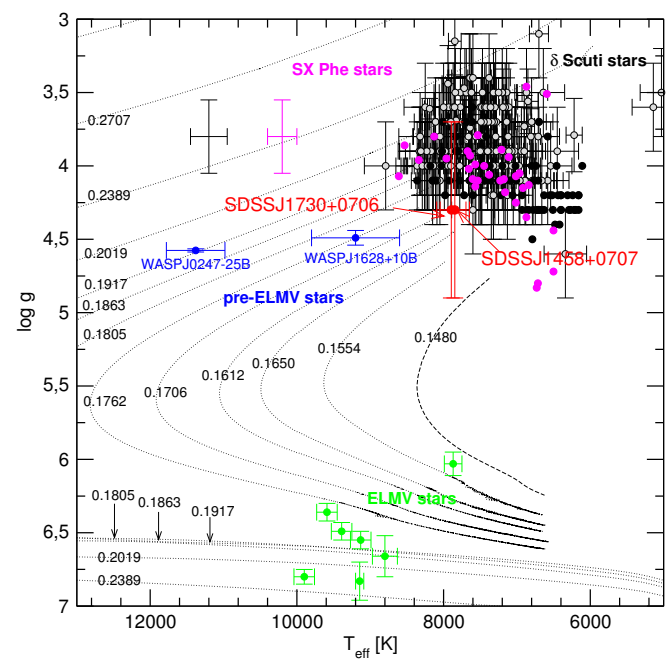

Fig. 5. Location of the stars SDSS J1730+0706 and SDSS J1458+0707 in the $T_{\text {eff }}-\log g$ plane, with samples of other classes of variable stars (see the text). The crosses on the left side indicate the error of the black and pink dots. The extremely short-lived stages during the $\mathrm{CNO}$ flashes episodes of $M_{\star}>0.1762 M_{\odot}$ are not shown in the figure.

Althaus, L. G., Miller Bertolami, M. M., \& Córsico, A. H. 2013, A\&A, 557, A19 Balona, L. A., \& Nemec, J. M. 2012, MNRAS, 426, 2413

Bell, K. J., Kepler, S. O., Montgomery, M. H., et al. 2015, in 19th European Workshop on White Dwarfs, eds. P. Dufour, P. Bergeron, \& G. Fontaine, ASP Conf. Ser., 493, 217

Bradley, P. A., Guzik, J. A., Miles, L. F., et al. 2015, AJ, 149, 68

Brown, W. R., Kilic, M., Allende Prieto, C., \& Kenyon, S. J. 2010, ApJ, 723, 1072

Brown, W. R., Kilic, M., Allende Prieto, C., \& Kenyon, S. J. 2012, ApJ, 744, 142

Brown, W. R., Kilic, M., Allende Prieto, C., et al. 2013, ApJ, 769, 66 Catelan, M., \& Smith, H. A. 2015, Pulsating Stars (Wiley-VCH)

Driebe, T., Schoenberner, D., Bloecker, T., \& Herwig, F. 1998, A\&A, 339, 123

Fauvaud, S., Sareyan, J.-P., Ribas, I., et al. 2010, A\&A, 515, A39

Gianninas, A., Kilic, M., Brown, W. R., et al. 2015, in 19th European Workshop on White Dwarfs, eds. P. Dufour, P. Bergeron, \& G. Fontaine, ASP Conf. Ser., 493, 481

Hermes, J. J., Montgomery, M. H., Winget, D. E., et al. 2012, ApJ, 750, L28

Hermes, J. J., Montgomery, M. H., Gianninas, A., et al. 2013a, MNRAS, 436, 3573

Hermes, J. J., Montgomery, M. H., Winget, D. E., et al. 2013b, ApJ, 765, 102

Jeffery, C. S., \& Saio, H. 2013, MNRAS, 435, 885

Kanaan, A., Kepler, S. O., \& Winget, D. E. 2002, A\&A, 389, 896

Kepler, S. O. 1993, Balt. Astron., 2, 515

Kilic, M., Brown, W. R., Allende Prieto, C., et al. 2011, ApJ, 727, 3

Kilic, M., Brown, W. R., Allende Prieto, C., et al. 2012, ApJ, 751, 141

Kilic, M., Hermes, J. J., Gianninas, A., \& Brown, W. R. 2015, MNRAS, 446, L26

Koester, D. 2010, Mem. Soc. Astron. It., 81, 921

Koester, D., Voss, B., Napiwotzki, R., et al. 2009, A\&A, 505, 441

Maxted, P. F. L., Anderson, D. R., Burleigh, M. R., et al. 2011, MNRAS, 418, 1156

Maxted, P. F. L., Serenelli, A. M., Miglio, A., et al. 2013, Nature, 498, 463

Maxted, P. F. L., Serenelli, A. M., Marsh, T. R., et al. 2014, MNRAS, 444, 208

Serenelli, A. M., Althaus, L. G., Rohrmann, R. D., \& Benvenuto, O. G. 2001, MNRAS, 325, 607

Uytterhoeven, K., Moya, A., Grigahcène, A., et al. 2011, A\&A, 534, A125

Zacharias, N., Finch, C. T., Girard, T. M., et al. 2012, VizieR Online Data Catalog: I/322 